\title{
Evaluation of Regional Water Resources
}

\author{
Wenmin Wang \\ School of North China Electric Power University, Baoding 071000, China \\ 18073186248@163.com
}

Keywords: Regional water resources, carrying capacity,evaluate model.

\begin{abstract}
To evaluate the regional water resources carrying capacity according to the importance of each related factor,we establish a model and sort out indicators that can characterize the ability of a region to provide clean water for its population. The water deficient ratio and utilization ratio of water resources are selected as the final evaluation indexes, which are of great practical value. We use the fuzzy membership function method, combined with the principal component analysis to construct a multi-index comprehensive evaluation index system.
\end{abstract}

\section{Introduction}

Water scarcity may lead to drought. Humans historically have suffered a lot from drought, which happens more frequently than other disasters. For example, in 1921 in Soviet Union over 5 million people perished from starvation due to drought, and in 2006, Sichuan, China, experienced its worst drought in modern times with nearly 8 million people and over 7 million cattle facing water shortages[1]. These droughts are closely related to rainfall. By searching the information, we found that, besides human activities, water scarcity also results from its geographical location, altitude, distance from the major river systems, and the vegetation coverage, etc. These factors will then affect the precipitation, snowfall and evaporation. Instead of using water immoderately until the drought, a good understanding of the water resources carrying capacity will be of great significance.

\section{Evaluation Model of Water Resources Carrying Capacity}

First, we establish the equation according to the balance between supply and demand of water resources. Then, we use the principal component analysis(PCA) to determine the weight of each parameter affecting the total water demand. And finally we develop the fuzzy comprehensive evaluation model to analyze the influence of each parameter on the water supply capacity.

The assumptions of the model are as follows:

Assuming that there are no accidents, such as large-scale infectious disease, war, natural disasters and so on.

Assuming that the industrial structure of the industrial and agricultural industries will not be changed for the policy.

Assuming that the total discharge of sewage in the region will not exceed a certain limit in the year of our study.

No consideration is given to the loss of water from the surface and ground water.

The runoff of the area is not less than the minimum base flow rate of the river, so that the area can meet the requirement of ecological water consumption.

First, we establish the equation according to the balance between supply and demand of water resources:

The model parameters and their meaning are shown in Table 1: 
Table 1 Model parameters

\begin{tabular}{|l|l|l|l|}
\hline Parameter & Meaning & Parameter & Meaning \\
\hline$W_{\text {total }}$ & $\begin{array}{l}\text { Regional water resources } \\
\text { including those that are } \\
\text { unavailable }\end{array}$ & $W_{P}, W_{I}, W_{A}, W_{E}, W_{\text {other }}$ & $\begin{array}{l}\text { water resources for residential, } \\
\text { industrial, agricultural, ecologic } \\
\text { and other purposes }\end{array}$ \\
\hline$W_{S}, W_{D}$ & Water supply; water demand & $W D R$ & Water deficient ratio \\
\hline$W_{R}$ & Return flow & $P$ & Regional population \\
\hline$W_{T}$ & $\begin{array}{l}\text { Water volume transferred from } \\
\text { other regions }\end{array}$ & $S$ & Agricultural irrigation area \\
\hline$W$ & $\begin{array}{l}\text { Amount of surface water and } \\
\text { groundwater }\end{array}$ & $R$ & $\begin{array}{l}\text { Regional gross industrial } \\
\text { production }\end{array}$ \\
\hline$\alpha$ & $\begin{array}{l}\text { Utilization ratio of water } \\
\text { resources }\end{array}$ & $C_{P}, C_{I}, C_{A}$ & $\begin{array}{l}\text { Water consumption per unit of } \\
\text { population, irrigation and } \\
\text { industrial production }\end{array}$ \\
\hline
\end{tabular}

$$
\begin{array}{lll}
W_{S}=W+W_{T}+W_{R} & \text { (1) } & W_{D}=W_{P}+W_{I}+W_{A}+W_{E}+W_{\text {other }} \\
W D R=\frac{W_{D}-W_{S}}{W_{D}}=1-\frac{W_{S}}{W_{D}} & \text { (3) } & \alpha=\frac{W_{S}}{W_{\text {total }}}
\end{array}
$$

Obviously, when the regional water supply is less than the demand( $\left.W_{S}<W_{D}, W D R>0\right)$, this shows that the regional clean water cannot support such a society on this scale. But if we increase water volume transferred from other regions and/or decrease water demand by saving water, $W D R$ will reduce. On the contrary, that is $W_{S} \geq W_{D}, W D R \leq 0$, this shows that the regional water resources can afford such a scale of society. Water supply and demand are in good condition.

Utilization ratio of water resources is the ratio of regional water supply and the total amount of water resources including those that are unavailable. It reflects the utilization of water resources. We divide water resources into three levels by utilization ratio:

$\alpha<30 \%$ : The society is in water-consuming pattern, water health is in good condition.

$30 \%<\alpha<50 \%$ : The society is changing to water-saving pattern, water health is under pressure.

$\alpha>50 \%$ : The society is in water-saving pattern,water health is deteriorated.

Secondly, we use principal component analysis to determine the weights:

The principal component analysis method is a statistical analysis method to change the original multiple variables into a few comprehensive indexes. It makes the contribution of each index to the

\begin{tabular}{|c|c|c|}
\hline Target layer & Criterion layer & Index layer \\
\hline \multirow{4}{*}{ Water supply index } & Regional water resources & Surface water and groundwater \\
\hline & \multirow{2}{*}{ Regional policy } & Water transferred from other regions \\
\hline & & Utilization ratio of water resources \\
\hline & Water pollution & Return flow \\
\hline \multirow{9}{*}{ Water demand index } & \multirow{4}{*}{ Resident life } & Urban population \\
\hline & & Annual water consumption per person in cities \\
\hline & & Rural population \\
\hline & & Annual water consumption per person in rural areas \\
\hline & \multirow{4}{*}{ Economic development } & Agricultural irrigation area \\
\hline & & Water consumption per unit of irrigation \\
\hline & & Gross industrial production \\
\hline & & Water consumption per unit of industrial production \\
\hline & Ecological condition & Ecological water demand \\
\hline
\end{tabular}
overall system as the weight. We make the analysis through SPSS. The evaluation system is divided into three layers: target layer, criterion layer and index layer. Indicators are shown in the Table 2:

Table 2 Each indicator in the evaluation system

Analysis steps are as follows: 
Step1: We use Altman's Z-Score standardize the raw data to eliminate the influence of dimension. Through Z- Score transformation, we calculate the mean value $(\bar{X})$ and standard deviation(S) of the variable data $(X)$, and obtain the normalized value $\left({ }^{X}\right)$. It can be represented as:

$$
x=\frac{(X-\bar{X})}{S}
$$

These normalized values are represented as $x_{1}, x_{2}, \ldots, x_{j}, \ldots$

Step 2: We use the SPSS13.0 software to form the correlation coefficient matrix $\mathbf{R}$, through principal component analysis method, each factor can be extracted from the correlation coefficient matrix, and the factor load matrix $\mathbf{F}$ is obtained. The weight of the corresponding factor is identified by the variance contribution degree, so that the weight set is obtained.

In order to determine the weight distribution of evaluation index, we establish the following regression equation according to the correlation coefficient matrix $\mathbf{R}$ and each column factor load vector $F_{k}$ :

$$
\mathbf{R} \times \overrightarrow{b_{k}}=\overrightarrow{F_{k}}
$$

Then we can obtain the principal component contribution matrix $\overrightarrow{b_{k}} \cdot{ }^{b_{k}}$ represents the principal component contribution of the $k$ th coefficient. The combination of $b_{m k}$ and the corresponding variance contribution $D_{k}$ is the weight of the $m$ th evaluation index.

Step 3: Finally, we normalize the weight matrix $\overrightarrow{B_{m}}$ to get the standard weight $W_{q j}$.

$$
\overrightarrow{B_{m}}=\sum_{k=1}^{n}\left|b_{m k}\right| \times D_{k}
$$

$$
\begin{aligned}
& k=1,2, \ldots, n,\left(n_{\text {is }}\right. \text { the number of principal components); } \\
& m=1,2, \ldots, p \text {, ( } p_{\text {is the number of indicators in the system). }}
\end{aligned}
$$

Finally, We then set up a fuzzy comprehensive evaluation model:

We adapt weighted fuzzy membership function as a comprehensive evaluation method. By using the principal component weighting method, we weight each index. In addition, taking the forward and reverse nature of the index into account, the fuzzy membership function method is used to quantify the indicators.

The positive index adopts the semi-rise trapezoidal fuzzy membership function:

$$
\phi_{j}= \begin{cases}1 \quad, \quad x_{j}=M_{j, \text { max }} \\ \frac{x_{j}-m_{j, \min }}{M_{j, \text { max }}-m_{j, \text { min }}}, & m_{j, \text { min }}<x_{j}<M_{j, \text { max }} \\ 0 \quad, & x_{j}=x_{j}=m_{j, \text { min }}\end{cases}
$$

The inverse index is used as a function of the fuzzy membership function:

$$
\phi_{j}= \begin{cases}1 \quad, \quad x_{j}=m_{j, \text { min }} \\ \frac{M_{j, \text { max }}-x_{j}}{M_{j, \text { max }}-m_{j, \text { min }}}, & m_{j, \text { min }}<x_{j}<M_{j, \text { max }} \\ 0 \quad, & x_{j}=M_{j, \text { max }}\end{cases}
$$

${ }^{x_{j}}$ : the standard data of the ${ }^{j}$ th index in the water supply/demand evaluation index system.

$M_{j, \max }, m_{j, \min }$ : the maximum/minimum value of the $j$ th index in a year respectively. 
$\phi_{j}$ : the membership degree of the index ${ }^{x_{j}}, 0 \leq \phi_{j} \leq 1$.

Then we can obtain the values of membership degree of each evaluation index $\left({ }^{\phi\left(x_{i j}\right)}\right)$ of water supply and demand in the study area.

Comprehensive evaluation index calculation model is represented as:

$$
Z_{q}=\sum_{j=1}^{p} W_{q j} \times \phi_{j}
$$

$Z_{q}$ : the regional water supply/demand index in $q_{\text {year, }} q=2001,2002, \ldots, 2014$

$p$ : number of indicators (when ${ }^{Z}{ }_{q}$ indicates a supply index, $p=4$; when ${ }^{Z_{q}}$ indicates a demand index, $p=7$;)

$W_{q j}$ : the weight of the $j$ th index in the water supply/demand evaluation index system in $q$ year.

We use PCA to weight $Z_{q}$, so the total water supply/demand evaluation index is calculated as follows:

$$
\begin{gathered}
Z=w_{2001} \cdot Z_{2001}+w_{2002} \cdot Z_{2002}+\cdots+w_{2014} \cdot Z_{2014} \\
w_{2001}, w_{2002}, \ldots, w_{2014} \text { : the weight of } Z_{2001}, Z_{2002}, \cdots, Z_{2014} \text {, respectively. }
\end{gathered}
$$

\section{Summary}

Our principal component weighting method pays more attention to the intrinsic link between the factors, so that the impact of the uncertainty on the results is greatly reduced, so it can be more objective to reflect the influence of each factor.It is integrated and comprehensive to study the relationship between regional supply and demand of clean water, combining principal component weighting method and fuzzy membership function method.

\section{References}

[1]. XIA Jun. Water security measure-The bearing capacity of water resources research and challenges. Haihe Water Resources, 2002, 2: 5-7. (in Chinese)

[2]. Li Cheng. System dynamics model of Suzhou water resources carrying capacity and its application[J]. Water Science and Engineering,2010,32:.

[3]. L. H. Feng,C. F. Huang. A Risk Assessment Model of Water Shortage Based on Information Diffusion Technology and its Application in Analyzing Carrying Capacity of Water Resources[J]. Water Resources Management,2008,225:.

[4]. He Yiqing, Weng Yijing. Evaluation on the coordinated development of urban resource environment and economy in Poyang Lake region [J]. resources science, 2012,03:502-509. 